\title{
Estudio de los impactos ambientales de los cruces de gasoductos con ríos vadeables
}

\author{
Morcillo, F¹., Arconada, B., Fernández, Y., Lacal, M. y Vadillo, L. ${ }^{2}$ \\ Instituto Geológico y Minero de España. Ríos Rosas, 23. 28003 Madrid. \\ ${ }^{1}$ f.morcillo@igme.es, ${ }^{2}$ 1.vadillo@igme.es
}

\begin{abstract}
RESUMEN
El río Cigüela y su afluente Valdepineda (Cuenca, España) fueron afectados por el cruce de un gasoducto, en Diciembre de 2001, mediante la técnica convencional "cruce subálveo" que consta de tres fases: instalación de la pista de trabajo, apertura de zanja y rehabilitación. Con el objetivo de evaluar el impacto de las distintas etapas de la obra en el medio acuático, se está llevando a cabo un estudio que se centra en el efecto de los sólidos en suspensión en las comunidades macrobentónicas e ícticas. Se presentan los resultados preliminares obtenidos en la fase de apertura de zanja, donde se observa un valor muy elevado de movilización de sólidos en suspensión (hasta $5000 \mathrm{mg} / \mathrm{l}$ ), alcanzando las mayores concentraciones en los 100 primeros metros aguas abajo del punto de cruce. Estos sólidos en suspensión podrían ser la causa del aumento significativo de la sedimentación de las partículas de tamaño comprendido entre 50-250 $\mu \mathrm{m}$ y 250-500 $\mu \mathrm{m}$ en los 30 primeros metros aguas abajo del punto de cruce. Por otro lado, no se han observado diferencias en el valor del índice BMWP' en las distintas estaciones de muestreo. Sin embargo, en la fase de rehabilitación, se encuentra una diferencia significativa en la frecuencia de distribución de algunas de las especies de peces presentes en el río, además se observa un claro efecto barrera para la ictiofauna al eliminar la pista de trabajo.
\end{abstract}

Palabras clave: gasoducto, cruce de ríos, control de impactos biológicos, rehabilitación, macroinvertebrados bentónicos, peces, sedimentos en suspensión.

\begin{abstract}
The Cigüela River and its tributary, Valdepineda (Cuenca, España) were affected by the crossing of a natural gas pipeline, in December 2001, by means of the conventional technique "open-cut water crossing" that consists of three different works: installation of the right-of-way, excavation and backfill of the pipeline trench, and restoration. In order to assess the impacts of these different activities on the aquatic environment, we are carrying out a study that is focused on the effects of suspended solids in the benthic and fish communities. Preliminary results of this study are presented on the excavation and backfill of the pipeline trench, that indicate that a very high value of suspended solids mobilisation is observed (up to $5000 \mathrm{mg} / \mathrm{l}$ ), reaching the higher concentrations in the first 100 meters downstream of the crossing site. These suspended solids might be responsible for the significant increase in the sedimentation of the particles of sizes 50-250 $\mu \mathrm{m}$ and 250-500 $\mu \mathrm{m}$ in the 30 first meters downstream of the crossing site. On the other hand, we have not found differences in the values of the BMWP'index at the different sampling sites. Nevertheless, in the restoration phase, there is a significant difference in the distribution frequency of some fish species that appear in the river and also a clear barrier effect is observed for fishes when eliminating the right-of-way.
\end{abstract}

Keywords: gas pipeline, water crossing, biological effects monitoring, recovery, benthic macro-invertebrates, fish, suspended sediments.

\section{INTRODUCCIÓN}

Las actividades constructivas que se realizan en ríos y arroyos llevan asociadas consigo un impacto sobre el ecosistema acuático. En la
Península Ibérica, una gran parte de estos ecosistemas han sufrido los efectos de la agricultura industrial que, con sus prácticas, han alterado la calidad y el caudal de las aguas. En los últimos años, la política energética de nuestro país 
está propiciando la construcción de gasoductos a lo largo y ancho de la geografía nacional y, como consecuencia del desarrollo de estas nuevas infraestructuras, se pueden producir cambios en los componentes físicos, biológicos y, en menor medida, en los componentes químicos de ríos y arroyos. Estas alteraciones y sus consecuencias se acentúan en arroyos vadeables, $a$ priori aparentemente más sensibles que los grandes ríos por la facilidad de acceso y de actuación en sus lechos.

A la hora de llevar a cabo este tipo de obras, existen diferentes metodologías de cruces. La mayor parte de ríos y arroyos vadeables en España, se cruzan con la técnica de construcción conocida como "cruce subálveo", que produce un considerable aumento de la concentración de sedimentos en la columna de agua en un corto período de tiempo durante la construcción. Esta técnica consta de tres fases: en primer lugar, la instalación de una pista de trabajo que conlleva el desbroce de la vegetación de ribera, el desmonte de los taludes y la instalación de una tubería (también llamada vaina) que canalice el río y mantenga su caudal; en segundo lugar, la apertura de una zanja, que requiere el desvío del caudal mediante un by-pass, la excavación de una zanja en el lecho del río, la instalación de una tubería y la deposición de materiales; en tercer lugar, la rehabilitación, que comporta el desmantelamiento de la pista de trabajo y la vaina, la construcción de una escollera y la restitución del lecho del río y la ribera.

Estas labores constructivas que afectan a los cursos fluviales pueden extenderse durante un largo período de tiempo y por tanto, producir un considerable incremento de sedimentos en suspensión, con las graves consecuencias que ello conlleva. Los efectos potenciales de las entradas de sedimentos y las perturbaciones en los componentes bióticos y abióticos de los ríos están bien documentados (Cordone y Kelley 1961; Chutter 1969; Barton 1977; Newcombe y MacDonald, 1991; Newcombe y Jensen 1996; Anderson et al., 1996). Más específicamente, otros autores han estudiado los efectos del incremento de la movilización de sedimentos debido a la construcción de gasoductos (Reid y Anderson, 1999) sobre las comunidades macrobentónicas (Tsui y McCart, 1981) y en las comunidades de peces y sus hábitats (McKinnon y Hnytka, 1988).

Considerando la cantidad de nuevos gasoductos que van a ser construidos en nuestro país, los desdoblamientos de los que ya están construidos y la necesidad de sustituir aquellos instalados por otros nuevos, creemos que es importante conocer cuales son los efectos potenciales para realizar una buena gestión de los recursos acuáticos. Por ello, y con la finalidad de evaluar los impactos potenciales de este tipo de obra, se está llevando a cabo un estudio que examina el conjunto de los cambios físicos y biológicos asociados con el cruce con estos ríos. Este estudio se ha diseñado específicamente para evaluar los efectos de construcción del gasoducto sobre la comunidad de macroinvertebrados bentónicos, la densidad de peces y su distribución, y las relaciones entre los cambios ecológicos y alteraciones en la granulometría del lecho del río. Para ello se han elegido las obras de construcción del gasoducto Tarancón-Cuenca-Fuentes en su cruce con el río Cigüela y su afluente Valdepineda (cuenca del Guadiana), en el término municipal de Horcajada de la Torre (provincia de Cuenca).

En este trabajo se presentan los resultados preliminares de un estudio que se desarrolla a lo largo de un ciclo anual desde unos días antes al inicio de las obras del gasoducto. Estos resultados se han obtenido en la fase de apertura de la zanja y, se presentan también los resultados relacionados con la presencia y abundancia de las distintas especies de peces antes y después de la fase de rehabilitación. Los objetivos globales de este proyecto son tres: en primer lugar evaluar los efectos de estas obras sobre los cursos hídricos permanentes y vadeables; en segundo lugar, proponer una serie de medidas preventivas y correctoras que minimicen estos impactos y, por último, identificar los indicadores ambientales adecuados que permitan aplicar una metodología sencilla al desarrollo de los planes de seguimiento de las medidas minimizantes en este tipo de proyectos. 


\section{ÁREA DE ESTUdIO}

El ámbito de estudio se sitúa en el Término Municipal de Horcajada de La Torre (provincia de Cuenca), España. El proyecto se desarrolla en dos tramos de aproximadamente 1.000 metros de longitud en el río Cigüela y su afluente Valdepineda, en la cuenca del Guadiana (Fig. 1). Estos ríos muy antropizados tienen en su mayor parte una anchura media de $1 \mathrm{~m}$ y una profundidad media de $80 \mathrm{~cm}$. En sus lechos hay un depósito de sedimentos finos de aproximadamente $30 \mathrm{~cm}$ de espesor, exceptuando las cuatro primeras estaciones del río Cigüela y la cuarta estación del Valdepineda, donde la profundidad es de unos $30 \mathrm{~cm}$ sobre arenas gruesas, gravas y cantos. Estos ríos lavan materiales yesíferos presentando, por tanto, un carácter marcadamente salino con un grado de mineralización superior a 2.000 $\mu \mathrm{S} / \mathrm{cm}$ y con un $\mathrm{pH}$ próximo a la neutralidad. Sus aguas se clasifican como sulfatadas cálcicas y ligeramente magnésicas según Shtchoukarev (Catalán La Fuente, 1981) en el Cigüela. La media de los valores de sólidos en suspensión encontrados a lo largo de un año de estudio son mayores en el río Valdepineda que en el Cigüela, 27.6 (5.4-273.5) frente a 7 (1.324.8) $\mathrm{mg} / 1$ (entre paréntesis el rango en el que

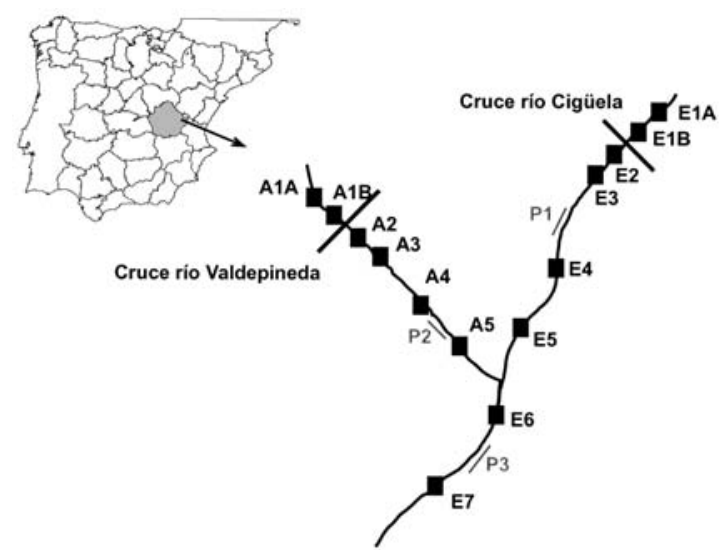

Figura 1. Esquema del área de estudio y de las distintas estaciones de muestreo (T.M. Horcajada de La Torre, Cuenca). Scheme of the location of the study area and sampling sites (Horcajada de La Torre municipality, Cuenca). se comprenden los datos). Lo mismo sucede con la turbidez, $18.8(0.88-51)$ frente a 8.6 (0.88-43.80) UNF. Los valores medios de caudal son similares para ambos ríos 86.4 (44.4142.7) 1/s para el río Cigüela y 73.2 (22.1129.3) 1/s para el Valdepineda.

La vegetación riparia está ausente, salvo algunos pies de chopo (Populus sp.) en las orillas, con lo que se ven reducidos, por una parte, los aportes de nutrientes al sistema, y por otra, la protección vegetativa de las mismas dejándolas más expuestas a la erosión. Esta vegetación de ribera ha sido sustituida por cultivos, los cuales llegan a sus orillas, observándose en ocasiones deslizamientos de las mismas como consecuencia de acopios de tierras. Las orillas son inestables, con morfología "en escalón", por lo tanto consideradas propensas a colapsar y sufrir erosión. Estos ríos son poco sinuosos debido al encauzamiento artificial, y esto les priva de una serie de ventajas, como la de responder mejor a los cambios de las fluctuaciones producidas, y como la de absorber la energía protegiendo al cauce de una excesiva erosión (FISRWG, 1998).

Ambos ríos presentan en su cauce una importante cantidad de helófitos y otros macrófitos emergentes y sumergidos que, además de actuar de trampa de sedimentos, sirven de alimento y refugio tanto para las aves como para la fauna acuática. Así, el carrizo (Phragmites communis) es la planta más abundante de la asociación vegetal dominante. Esta vegetación está constituida además por plantas herbáceas sólidamente asentadas y enraizadas en el agua pero que crecen fuera de ella. Al carrizo se asocian frecuentemente eneas o espadañas (Typha latifolia) y diferentes especies de juncos. También aparecen pies dispersos de masiega (Cladium mariscus). El fondo aparece tapizado por extensas praderas de plantas sumergidas de diferentes especies, aunque en nuestros tramos de estudio predomina el carófito Chara vulgaris. En primavera, emergen de esta vegetación subacuática plantas con flores entre las que sobresalen los ranúnculos (Ranunculus sp.) (obs. pers.). 


\section{MATERIAL Y MÉTODOS}

La obra de apertura de zanja se realizó en los ríos Valdepineda y Cigüela el 11 de diciembre de 2001. El día 10 de diciembre se realizaron los muestreos iniciales (fase de prezanja). El mismo día de la apertura de zanja se tomaron muestras de sólidos en suspensión durante la afección al lecho de los ríos (fase de zanja). Las obras finalizaron el 19 de diciembre, y el 21 de diciembre se realizaron los muestreos posteriores (fase de postzanja). Entre el 19 y el 22 de febrero de 2002, se realizó la restauración de ambos ríos con la retirada de la pista de trabajo. Los muestreos de ictiofauna se realizaron unos días antes del comienzo de las obras de construcción de la zanja, y en los períodos intermedios de todas las fases de obra para evitar los impactos directos sobre los peces de las actividades realizadas en el lecho del río.

Se seleccionaron 14 estaciones de muestreo a lo largo de los ríos Cigüela y Valdepineda (Fig. 1). De ellas, dos estaciones se sitúan aguas arriba del punto de cruce del gasoducto en cada uno de los ríos y el resto aguas abajo. Las dos últimas (E6 y E7) se sitúan aguas abajo de la zona de confluencia de ambos ríos. En el río Cigüela, las estaciones son: E1A, E1B (127 y 50 m aguas arriba del cruce con el gasoducto, respectivamente), E2, E3, E4, E5, E6 y E7 (30, 70, 252, 407,700 y $870 \mathrm{~m}$ aguas abajo del cruce con el gasoducto, respectivamente). En el Valdepineda son: A1A, A1B (127 y $50 \mathrm{~m}$ aguas arriba del cruce con el gasoducto, respectivamente), A2, A3, A4 y A5 (30, 252, 407 y $507 \mathrm{~m}$ aguas abajo del cruce con el gasoducto, respectivamente).

En las fases de prezanja y postzanja se realizaron, en cada una de las estaciones de muestreo, determinaciones de parámetros físicoquímicos in situ: temperatura $\left({ }^{\circ} \mathrm{C}\right), \mathrm{pH}$, conductividad $(\mathrm{mS} / \mathrm{cm})$, oxígeno disuelto $(\mathrm{mg} / 1$ y $\%$ saturación) y turbidez. La temperatura y el $\mathrm{pH}$ se midieron con un pHmetro portátil Hanna HI $9025 \mathrm{C}$, la conductividad con un medidor multirango Hanna HI 9033, el oxígeno disuelto con un oxímetro portatil OXI 330 y la turbidez con un medidor Hanna HI 93703. Para el análisis de los sólidos en suspensión se tomaron en campo muestras en botes de plástico de $500 \mathrm{ml}$ de capacidad y posteriormente se midieron en el laboratorio por el método gravimétrico PTE-AG020. Para el cálculo de caudales se realizaron por estación un transecto perpendicular a la corriente con un mínimo de 8 medidas de profundidad y de velocidad de corriente.

Para el análisis granulométrico del sedimento del río se tomaron 3 réplicas para cada estación y muestreo y se separaron en tres fracciones: 50-250 $\mu \mathrm{m}, 250-500 \mu \mathrm{m}$ y $500-1000 \mu \mathrm{m}$. Las muestras se tomaron con cilindros de metacrilato de $6 \mathrm{~cm}$ de diámetro que se clavaron en el lecho del río extrayendo los 5 primeros $\mathrm{cm}$. Las fracciones se separaron en laboratorio con tamices calibrados de 50,250, 500 y $1000 \mu \mathrm{m}$, se secaron a $105^{\circ} \mathrm{C}$ durante 24 horas en una estufa de secado universal de rango 40 a $250{ }^{\circ} \mathrm{C}$, y se pesaron en una balanza analítica de tres decimales (precisión $0.001 \mathrm{gr}$ ). Los valores medios $\left(\mathrm{gr} / \mathrm{cm}^{2}\right)$ obtenidos para cada fracción en cada estación durante las fases de prezanja y postzanja son comparados con un test de la t. Estos datos se contrastan con los valores de la materia en suspensión obtenidos durante la construcción de la zanja. Durante la apertura de la zanja, se tomaron muestras de los sólidos en suspensión simultáneamente en todas las estaciones para comprobar la velocidad de avance de la pluma. El tiempo de toma de las muestras se realizó a los $5,10,30,45,60,120,180$ y 240 minutos después de comenzar los trabajos de excavación de la zanja en el punto de cruce. La materia en suspensión se cuantifica por el método gravimétrico con secado a $105^{\circ} \mathrm{C}$ (PTE-AG-020).

La recolección de los macroinvertebrados bentónicos presentes en todos los hábitats en cada punto de muestreo se llevó a cabo con una red Kicker de $250 \mu \mathrm{m}$ de luz de malla; las muestras se fijaron en alcohol de $70^{\circ}$. Entre los puntos E2, E3 y A2, A3 se ha recogido una sola muestra de macrobentos por muestreo con el fin de evitar en lo posible la perturbación del medio durante la recogida del material debido a la proximidad de las estaciones. Las muestras se identificaron hasta el nivel de familia mediante observaciones 
a la lupa Kyowa 40X, con el apoyo de claves y guías taxonómicas. Posteriormente se ha calculado el índice de macroinvertebrados BMWP' (Alba-Tercedor y Sánchez-Ortega, 1988). Para el muestreo de la ictiofauna se realizaron pescas eléctricas a lo largo de tres transectos longitudinales: P1 (50 m entre E3 y E4), P2 (50 m entre A3 y A4) y P3 (100 m entre E6 y E7). El método utilizado para la captura de los ejemplares fue la pesca eléctrica, con un motor generador HONDA EX350, montado sobre una mochila. La potencia del motor es de $300 \mathrm{~W}$ y la tensión
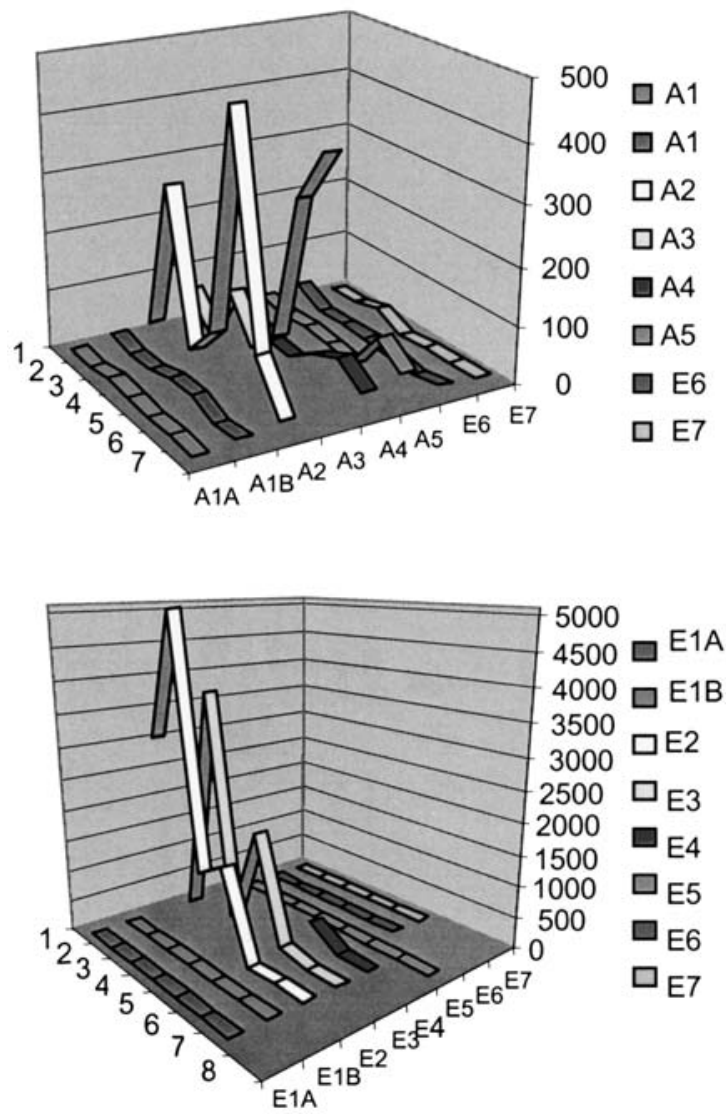

Figura 2. Movilización de sedimentos durante la fase de apertura de la zanja. En el eje Y se representa el intervalo de toma de muestras desde el inicio de los trabajos de excavación: 1 (5’), 2(10’), 3 (30’), 4 (45’), 5 (60’), 6 (120’), 7 (180’) y 8 (240'). Datos en mg/l. Suspended sediments mobilization during pipeline trench opening. Sample time intervals since beginning of trench opening is shown in the Y-axis: 1 (5'), 2(10'), 3 (30'), 4 (45'), 5 (60'), 6 (120'), 7 (180') y 8 (240'). Data in $\mathrm{mg} / \mathrm{l}$. de salida se puede regular: 150/200/300 V. Siempre se trabajó con intensidades de corriente lo suficientemente pequeñas como para evitar mortandades innecesarias en las poblaciones. Para comparar las diferencias en la frecuencia de distribución del número de individuos de peces de una determinada especie se ha calculado el valor de Chi-cuadrado.

\section{RESULTADOS}

El análisis de los sólidos en suspensión realizados antes del inicio de las obras en todas las estaciones muestreadas está por debajo de los $9 \mathrm{mg} / \mathrm{l}$ para el río Cigüela. Sin embargo, el Valdepineda es muy irregular, habiéndose encontrado un valor medio de $65.7 \mathrm{mg} / \mathrm{l}$ en un rango comprendido entre 10.6 (en A4) y $273 \mathrm{mg} / 1$ (en A3).

La pluma formada por el sedimento en suspensión movilizado una vez iniciada la construcción de la zanja del gasoducto en el río Valdepineda tardó unos 10 minutos en alcanzar los primeros $30 \mathrm{~m}$ aguas abajo del punto de cruce (A2), llegando a valores de $284 \mathrm{mg} / \mathrm{l}$, y unas 2 horas hasta alcanzar A5 (84 mg/l), aproximadamente a $500 \mathrm{~m}$ aguas abajo del punto de cruce (Fig. 2). No se ha detectado afección sobre las dos estaciones fijadas aguas arriba del punto de cruce (A1A y A1B) ni sobre las situadas a partir de los $600 \mathrm{~m}$ del punto de cruce del río Valdepineda (E6 y E7), al menos en las dos horas posteriores a la realización de las obras. Con relación al río Cigüela, se detecta un considerable aumento de los sólidos en suspensión en los primeros $30 \mathrm{~m}$ aguas abajo del punto de cruce (E2) a los 5 minutos de iniciarse la obra $(3016 \mathrm{mg} / \mathrm{l})$ y a $\operatorname{los} 70 \mathrm{~m}(\mathrm{E} 3)$ a $\operatorname{los} 10$ minutos. Debido a un error de muestreo no se dispone de datos de la primera hora en la estación situada a $250 \mathrm{~m}$ aguas abajo (E4). Sin embargo, los datos horarios recogidos indican un valor máximo (339 mg/l) al cabo de 1 hora después de iniciada la obra. Al igual que en el Valdepineda, no se ha detectado afección sobre las dos estaciones fijadas aguas arriba del punto de cruce (E1A y E1B) ni sobre las situadas a partir de los $400 \mathrm{~m}$ 
del punto de cruce del río Cigüela (E5, E6 y E7), al menos en las dos horas posteriores a la realización de las obras.

Unos días después de finalizar la construcción, cinco en el caso del río Valdepineda y dos en el del Cigüela, los valores de los sólidos en suspensión se recuperaron hasta los iniciales, con valores medios de 17 y $4.4 \mathrm{mg} / 1$ para los ríos Valdepineda y Cigüela, respectivamente.

Toda esta movilización de los sólidos en suspensión se refleja en algunos de los resultados obtenidos del estudio granulométrico del sedimento (Tabla 1). Así, en la fase posterior a la apertura de la zanja se observa un aumento sig-

Tabla 1. Análisis estadístico de la granulometría del sedimento del lecho del río antes (peso prezanja) y después de la construcción de la zanja (peso postzanja). Las diferencias significativas (t-Student) halladas en las estaciones de muestreo se señalan en negrita. Datos en $\mathrm{g} / \mathrm{cm}^{2}$. Statistical analysis of sediment granulometry from the streambed before and after trench construction. Significant values (t-Student) in each sampling site are shown in bold. Data in $\mathrm{g} / \mathrm{cm}^{2}$.

\begin{tabular}{|c|c|c|c|c|c|}
\hline & Peso prezanja & Peso poszanja & t-value & df & $\mathbf{p}$ \\
\hline A1A $(50-250 \mu \mathrm{m})$ & 10.51 & 11.11 & -0.381 & 4 & 0.723 \\
\hline $\mathrm{A} 1 \mathrm{~A}(250-500 \mu \mathrm{m})$ & 1.57 & 1.44 & 0.381 & 4 & 0.723 \\
\hline $\mathrm{A} 1 \mathrm{~A}(500-1000 \mu \mathrm{m})$ & 1.08 & 0.97 & 0.729 & 4 & 0.506 \\
\hline A1B $(50-250 \mu \mathrm{m})$ & 6.84 & 49.52 & -1.690 & 4 & 0.166 \\
\hline A1B $(250-500 \mu \mathrm{m})$ & 1.11 & 39.22 & -1.254 & 4 & 0.278 \\
\hline A1B $(500-1000 \mu \mathrm{m})$ & 1.31 & 40.63 & -1.323 & 4 & 0.256 \\
\hline A2 $(50-250 \mu \mathrm{m})$ & 13.13 & 30.95 & -7.625 & 4 & 0.002 \\
\hline A2 $(250-500 \mu \mathrm{m})$ & 1.70 & 14.21 & -5.753 & 4 & 0.005 \\
\hline A2 $(500-1000 \mu \mathrm{m})$ & 1.05 & 16.65 & -13.896 & 4 & 0.000 \\
\hline A3 $(50-250 \mu \mathrm{m})$ & 10.79 & 6.79 & 0.610 & 4 & 0.575 \\
\hline A3 $(250-500 \mu \mathrm{m})$ & 3.59 & 3.33 & 0.073 & 4 & 0.945 \\
\hline A3 $(500-1000 \mu \mathrm{m})$ & 4.09 & 3.47 & 0.141 & 4 & 0.895 \\
\hline A4 $(50-250 \mu \mathrm{m})$ & 48.39 & 22.24 & 0.967 & 4 & 0.388 \\
\hline A4 $(250-500 \mu \mathrm{m})$ & 37.88 & 4.78 & 1.064 & 4 & 0.347 \\
\hline A4 $(500-1000 \mu \mathrm{m})$ & 37.42 & 4.47 & 1.050 & 4 & 0.353 \\
\hline A5 $(50-250 \mu \mathrm{m})$ & 11.91 & 14.47 & -0.721 & 4 & 0.511 \\
\hline A5 $(250-500 \mu \mathrm{m})$ & 1.37 & 3.12 & -2.094 & 4 & 0.104 \\
\hline A5 $(500-1000 \mu \mathrm{m})$ & 0.61 & 1.26 & -2.567 & 4 & 0.062 \\
\hline E6 $\quad(50-250 \mu \mathrm{m})$ & 18.17 & 7.81 & 2.610 & 4 & 0.059 \\
\hline E6 $(250-500 \mu \mathrm{m})$ & 3.91 & 1.36 & 3.408 & 4 & 0.027 \\
\hline E6 $(500-1000 \mu \mathrm{m})$ & 2.69 & 1.40 & 1.513 & 4 & 0.205 \\
\hline E7 $\quad(50-250 \mu \mathrm{m})$ & 1.71 & 9.80 & -1.561 & 4 & 0.194 \\
\hline E7 $(250-500 \mu \mathrm{m})$ & 1.09 & 4.56 & -2.067 & 4 & 0.108 \\
\hline E7 $(500-1000 \mu \mathrm{m})$ & 2.93 & 5.99 & -1.394 & 4 & 0.236 \\
\hline $\mathrm{E} 1 \mathrm{~A}(50-250 \mu \mathrm{m})$ & 5.47 & 11.62 & -1.317 & 4 & 0.258 \\
\hline E1A $(250-500 \mu \mathrm{m})$ & 1.58 & 3.88 & -1.358 & 4 & 0.246 \\
\hline E1A $(500-1000 \mu \mathrm{m})$ & 0.59 & 1.18 & -3.694 & 4 & 0.021 \\
\hline E1B $(50-250 \mu \mathrm{m})$ & 14.31 & 7.32 & 4.545 & 4 & 0.010 \\
\hline E1B $(250-500 \mu \mathrm{m})$ & 2.04 & 1.60 & 0.835 & 4 & 0.451 \\
\hline E1B $(500-1000 \mu \mathrm{m})$ & 1.34 & 1.45 & -0.328 & 4 & 0.759 \\
\hline E2 $(50-250 \mu \mathrm{m})$ & 11.47 & 79.32 & -4.444 & 4 & 0.011 \\
\hline E2 $(250-500 \mu \mathrm{m})$ & 15.11 & 51.45 & -4.577 & 4 & 0.010 \\
\hline E2 $(500-1000 \mu \mathrm{m})$ & 14.72 & 12.14 & 0.339 & 4 & 0.751 \\
\hline E3 $(50-250 \mu \mathrm{m})$ & 12.53 & 14.61 & -0.879 & 4 & 0.429 \\
\hline E3 $(250-500 \mu \mathrm{m})$ & 22.15 & 8.70 & 1.498 & 4 & 0.209 \\
\hline E3 $(500-1000 \mu \mathrm{m})$ & 18.31 & 9.35 & 1.239 & 4 & 0.283 \\
\hline E4 $(50-250 \mu \mathrm{m})$ & 10.61 & 9.79 & 0.430 & 4 & 0.690 \\
\hline E4 $(250-500 \mu \mathrm{m})$ & 7.94 & 6.80 & 0.773 & 4 & 0.483 \\
\hline E4 $(500-1000 \mu \mathrm{m})$ & 4.63 & 5.72 & -0.945 & 4 & 0.398 \\
\hline E5 $(50-250 \mu \mathrm{m})$ & 14.62 & 11.64 & 0.968 & 4 & 0.388 \\
\hline
\end{tabular}


Tabla 1. (Continuación.)

\begin{tabular}{|c|c|c|c|c|c|c|}
\hline E5 & $(250-500 \mu \mathrm{m})$ & 3.57 & 4.81 & -8.008 & 4 & 0.001 \\
\hline E5 & $(500-1000 \mu \mathrm{m})$ & 2.20 & 2.70 & -2.023 & 4 & 0.113 \\
\hline E6 & $(50-250 \mu \mathrm{m})$ & 18.17 & 5.96 & 2.911 & 4 & 0.044 \\
\hline E6 & $(250-500 \mu \mathrm{m})$ & 3.91 & 0.77 & 4.123 & 4 & 0.015 \\
\hline E6 & $(500-1000 \mu \mathrm{m})$ & 2.69 & 0.71 & 2.559 & 4 & 0.063 \\
\hline E7 & $(50-250 \mu \mathrm{m})$ & 1.71 & 1.69 & 0.016 & 4 & 0.988 \\
\hline E7 & $(250-500 \mu \mathrm{m})$ & 1.09 & 0.69 & 0.441 & 4 & 0.682 \\
\hline E7 & $(500-1000 \mu \mathrm{m})$ & 2.93 & 0.97 & 0.917 & 4 & 0.411 \\
\hline
\end{tabular}

nificativo en la sedimentación de las partículas de tamaño 50-250 $\mu \mathrm{m}, 250-500 \mu \mathrm{m}$ y $500-1000$ $\mu \mathrm{m}$ y en los primeros 30 metros aguas abajo del punto de cruce del gasoducto en el Valdepineda (A2) y de tamaño $50-250 \mu \mathrm{m}$ y $250-500 \mu \mathrm{m}$ en el Cigüela (E2) (Fig. 3). También en E5 se observa un ligero incremento en la sedimentación de partículas de tamaño $250-500 \mu \mathrm{m}$. Por el contrario, las diferencias significativas en la composición granulométrica de los sedimentos

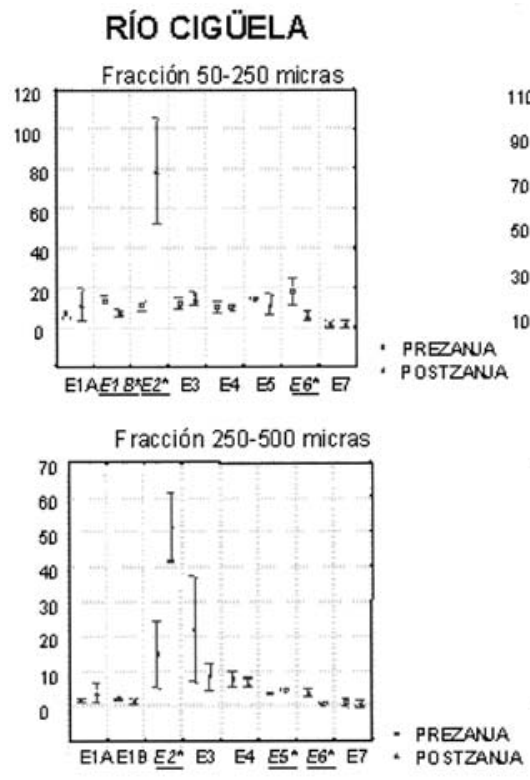

RIO VALDEPINEDA
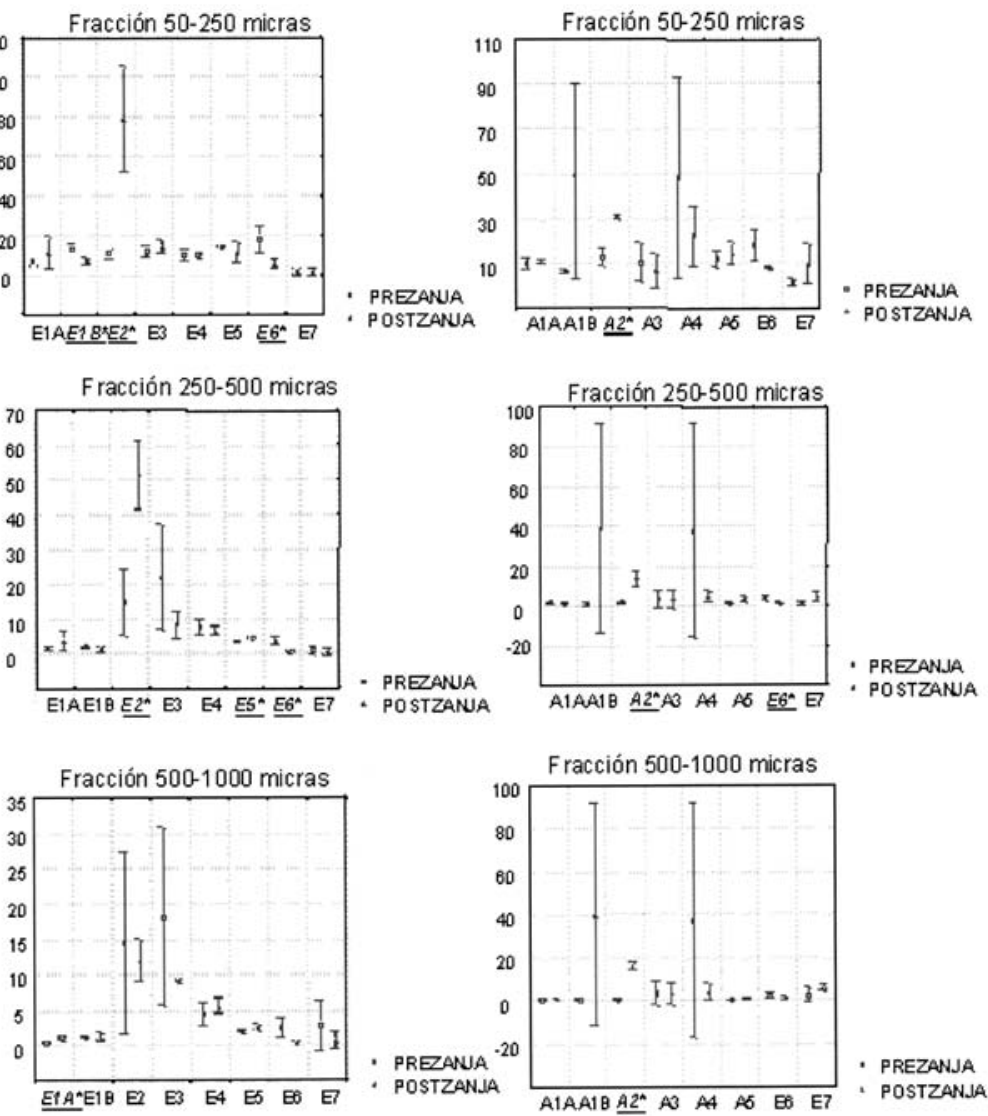

Figura 3. Análisis estadístico de la granulometría del sedimento del lecho del río antes y después de la construcción de la zanja. Las diferencias significativas halladas en las estaciones de muestreo se señalan en cursiva y subrayadas. Datos en ordenadas en $\mathrm{g} / \mathrm{cm}^{2}$. Statistical analysis of sediment granulometry from the streambed before and after trench opening. Significant values in each sampling site are shown in italics and underlined. Data in Y-axis in $\mathrm{g} / \mathrm{cm}^{2}$. 
Tabla 2. Resultados de los análisis físico-químicos realizados en las estaciones de los ríos Cigüela y Valdepineda antes (1) y después (2) de la construcción de la zanja. Abreviaturas: Cond: conductividad; O.D.: oxígeno disuelto; T: turbidez; SST: sólidos en suspensión totales; -: sin caudal circulante. Results of the physical and chemical analyses performed at the sites in the Cigüela and Valdepineda Rivers before (1) and after (2) trench construction. Abbreviations: Cond: conductivity; O.D.: dissolved oxygen; T: turbidity; SST: total suspended solid; -: without flow.

\begin{tabular}{|c|c|c|c|c|c|c|c|c|c|c|c|c|c|c|c|c|}
\hline & \multicolumn{2}{|c|}{ pH } & \multicolumn{2}{|c|}{$\mathrm{T}\left({ }^{\circ} \mathrm{C}\right)$} & \multicolumn{2}{|c|}{ Cond. (ms/cm) } & \multicolumn{2}{|c|}{ O.D. (mg/L) } & \multicolumn{2}{|c|}{ O.D. (\%) } & \multicolumn{2}{|c|}{ T (UNF) } & \multicolumn{2}{|c|}{ SST (mg/l) } & \multicolumn{2}{|c|}{ Caudal } \\
\hline & 1 & 2 & 1 & 2 & 1 & 2 & 1 & 2 & 1 & 2 & 1 & 2 & 1 & 2 & 1 & 2 \\
\hline E1A & 7.9 & 7.9 & 3.6 & 1.7 & 3.66 & 3.73 & 10.98 & 10.36 & 91.1 & 83.0 & 2.06 & 3.27 & 8.60 & 2.40 & - & - \\
\hline E1B & 8.0 & 8.0 & 3.5 & 1.7 & 3.56 & 3.73 & 10.92 & 10.50 & 90.7 & 82.3 & 2.13 & 3.48 & 8.60 & 2.90 & - & - \\
\hline E2 & 8.0 & 8.0 & 3.5 & 1.7 & 3.60 & 3.60 & 10.88 & 10.60 & 90.1 & 83.3 & 1.49 & 4.86 & 8.80 & 0.60 & 50.1 & 43.8 \\
\hline E3 & 8.0 & 8.0 & 3.5 & 1.7 & 3.53 & 3.80 & 11.06 & 10.80 & 92.0 & 85.3 & 1.25 & 5.88 & 5.40 & 3.60 & 44.4 & 50.5 \\
\hline E4 & 7.9 & 7.9 & 3.8 & 1.6 & 3.60 & 3.50 & 11.63 & 11.20 & 97.6 & 87.0 & 0.88 & 2.97 & 8.30 & 1.70 & 71.5 & 47.2 \\
\hline E5 & 7.9 & 8.0 & 3.4 & 1.7 & 3.56 & 3.70 & 11.86 & 11.00 & 98.3 & 86.3 & 3.06 & 3.33 & 7.40 & 2.80 & 93.1 & 100.4 \\
\hline E6 & 7.9 & 7.9 & 4.6 & 1.2 & 3.70 & 3.26 & 11.23 & 10.91 & 96.0 & 84.1 & 4.78 & 11.01 & 7.60 & 15.40 & 129.3 & 155.4 \\
\hline E7 & 8.2 & 8.1 & 4.8 & 3.0 & 3.64 & 3.33 & 10.86 & 9.99 & 93.0 & 81.4 & 5.57 & 9.39 & 6.00 & 5.80 & 118.3 & 121.9 \\
\hline A1A & 8.1 & 8.1 & 7.4 & 7.6 & 3.20 & 3.50 & 10.04 & 8.80 & 92.3 & 81.3 & 8.65 & 11.56 & 24.90 & 16.10 & 97.2 & 102.1 \\
\hline A1B & 8.1 & 8.1 & 7.4 & 7.7 & 3.20 & 3.59 & 10.28 & 8.53 & 94.2 & 79.6 & 19.29 & 12.99 & 50.80 & 8.20 & - & - \\
\hline A2 & 7.8 & 8.1 & 7.2 & 7.0 & 3.24 & 3.56 & 10.49 & 9.70 & 96.0 & 88.0 & 8.06 & 18.44 & 21.40 & 26.20 & 49.3 & - \\
\hline A3 & 8.1 & 8.1 & 7.1 & 6.7 & 3.20 & 3.50 & 10.39 & 9.83 & 95.0 & 89.0 & 50.00 & 22.77 & 273.5 & 17.60 & - & . \\
\hline A4 & 8.2 & 8.0 & 6.7 & 6.4 & 3.03 & 3.50 & 11.05 & 1.10 & 99.4 & 90.6 & 7.83 & 18.77 & 10.60 & 12.50 & 89.9 & 76.5 \\
\hline A5 & 8.1 & 8.0 & 6.6 & 6.2 & 3.10 & 3.40 & 10.96 & 9.83 & 98.6 & 88.0 & 5.98 & 17.43 & 13.10 & 21.40 & 80.8 & 81.3 \\
\hline
\end{tabular}

del lecho del río en las estaciones E1A, E1B, E5 y E6 obtenidas, no parecen explicarse teniendo en cuenta los valores de los sólidos en suspensión obtenidos durante la fase de ejecución de la obra. No se ha encontrado correlación alguna entre los datos de sólidos en suspensión y caudal en el río Valdepineda, ni antes (rPearson $=-0.15 ; \mathrm{p}=0.8$ ) ni después (rPearson $=0.07 ; \mathrm{p}=0,9)$ de la realización de la zanja. En cuanto al río Cigüela, antes de la realización de la zanja no se ha encontrado correlación entre sólidos en suspensión y caudal (rPearson= $-0.08 ; \mathrm{p}=0.957$ ), pero después de la realización de la misma es encuentra una correlación positiva significativa (rPearson $=0.86 ; 0.005)$.

Con respecto a los parámetros físico-químicos medidos no se observan diferencias entre los valores obtenidos antes y después de la realización de la zanja (Tabla 2). Sin embargo, durante la realización de la misma se tomaron, con la misma metodología, las correspondientes medidas de temperatura, $\mathrm{pH}$, conductividad, oxígeno disuelto y turbidez en los puntos E2 y A2. Comparando estos resultados con los obte- nidos en los mismos puntos antes y después de la obra, encontramos únicamente diferencias en la turbidez, $207 \mathrm{UNF}$ en A2 frente a $8.06 \mathrm{y}$ 18.44 (antes y después) y 435 UNF en E2 frente a 1.49 y 4.86 (antes y después).

Respecto a los macroinvertebrados, se realiza una comparativa de los taxa presentes antes y después de la construcción de la zanja en ambos ríos (Tablas 3 y 4) donde, a nivel de familia, fueron encontradas un total de 54, 46 pertenecientes al río Valdepineda y 45 al río Cigüela. El porcentaje de familias de insectos presentes en ambos ríos son muy superiores al resto de familias: insectos $(76.1 \%$ y $82 \%)$, gasterópodos (14.3\% y $9 \%)$, oligoquetos $(2.4 \%$ y $3 \%)$, bivalvos $(2.4 \%$ y $3 \%)$ y nemátodos $(2.4 \%$ y $0 \%)$, siendo el primer valor el perteneciente al río Valdepineda y el segundo al río Cigüela.

En el río Valdepineda se determinó un mayor número de familias pertenecientes a Odonata $\mathrm{y}$ Trichoptera, mientras que en el río Cigüela esto ocurrió con Plecoptera y Efemeroptera. Familias encontradas únicamente en el río Valdepineda son: Aeschnidae, Cordulegasteridae y Libellulidae 
Tabla 3. Listado de taxa de macroinvertebrados que aparecen en el río Valdepineda. Abreviaturas: L. Larvas; N: ninfas; A: adultos. List of macroinvertebrates' taxa found in the Valdepineda River. Abbreviations: L. Larvae; N: nymphs; A: adults.

\begin{tabular}{|c|c|c|c|c|c|c|c|c|c|c|}
\hline \multirow{2}{*}{$\frac{\text { Momentos }}{\text { Puntos de muestreo }}$} & \multicolumn{5}{|c|}{1} & \multicolumn{5}{|c|}{2} \\
\hline & $\mathbf{A 1 A}$ & A1B & A2-A3 & A4 & A5 & A1A & A1B & A2-A3 & A4 & A5 \\
\hline O.TRICLADOS & & & & & & & & & $\mathrm{X}$ & \\
\hline Cl. NEMATODOS & & & & $\mathrm{X}$ & & & & & & \\
\hline Cl. OLIGOQUETOS & & & & & $\mathrm{X}$ & & & & & \\
\hline F. HYDROBIIDAE & & & & $\mathrm{X}$ & & & & & & \\
\hline F. VALVATIDAE & $\mathrm{X}$ & & & & & & & & & \\
\hline F. ANCYLIDAE & $X$ & $X$ & $X$ & $\mathrm{X}$ & & $X$ & & $X$ & $\mathrm{X}$ & $\mathrm{X}$ \\
\hline F. PHYSIDAE & $X$ & $\mathrm{X}$ & $X$ & & $\mathrm{X}$ & & $X$ & $\mathrm{X}$ & & $\mathrm{X}$ \\
\hline F. LYMNAEIDAE & $\mathrm{X}$ & $X$ & $X$ & & $\mathrm{X}$ & $\mathrm{X}$ & $\mathrm{X}$ & $\mathrm{X}$ & & $\mathrm{X}$ \\
\hline F. SPHAERIIDAE & $\mathrm{X}$ & & & & $\mathrm{X}$ & $\mathrm{X}$ & $\mathrm{X}$ & & & $\mathrm{X}$ \\
\hline Cl. OSTRACODOS & & & $\mathrm{X}$ & & $\mathrm{X}$ & & & $\mathrm{X}$ & $\mathrm{X}$ & \\
\hline F. GAMMARIDAE & $\mathrm{X}$ & $X$ & $X$ & $\mathrm{X}$ & $\mathrm{X}$ & $X$ & $X$ & $\mathrm{X}$ & $\mathrm{X}$ & $\mathrm{X}$ \\
\hline O. HIDRACARINA & & & & $\mathrm{X}$ & & & & & $\mathrm{X}$ & $\mathrm{X}$ \\
\hline F. CAENIDAE & $X$ & $X$ & $X$ & & & & $X$ & $X$ & & $\mathrm{X}$ \\
\hline F. BAETIDAE & $\mathrm{X}$ & & $X$ & $\mathrm{X}$ & $\mathrm{X}$ & $\mathrm{X}$ & $\mathrm{X}$ & $\mathrm{X}$ & $\mathrm{X}$ & $\mathrm{X}$ \\
\hline F. NEMOURIDAE & & $\mathrm{X}$ & & & & & $\mathrm{X}$ & $\mathrm{X}$ & $\mathrm{X}$ & \\
\hline F.CAPNIIDAE & $\mathrm{X}$ & & & & & & & & & \\
\hline F. PERLODIDAE & & $\mathrm{X}$ & & $\mathrm{X}$ & & & & $\mathrm{X}$ & & $\mathrm{X}$ \\
\hline F. PERLIDAE & & & & & & $X$ & & & $\mathrm{X}$ & \\
\hline F. CALOPTERYGIDAE & $\mathrm{X}$ & $\mathrm{X}$ & & & & $\mathrm{X}$ & $\mathrm{X}$ & $\mathrm{X}$ & & $\mathrm{X}$ \\
\hline F. PLATYCNEMIDIDAE & $\mathrm{X}$ & & & & & & $\mathrm{X}$ & $\mathrm{X}$ & & $\mathrm{X}$ \\
\hline F. COENAGIONIDAE & $\mathrm{X}$ & $\mathrm{X}$ & $\mathrm{X}$ & & $\mathrm{X}$ & $\mathrm{X}$ & $\mathrm{X}$ & $\mathrm{X}$ & $\mathrm{X}$ & $\mathrm{X}$ \\
\hline F. AESCHNIDAE & & & & & $\mathrm{X}$ & & & & & $\mathrm{X}$ \\
\hline F. CORDULEGASTERIDAE & & & & & & & & & & $\mathrm{X}$ \\
\hline F. LIBELLULIDAE & & $\mathrm{X}$ & & & & & & & & \\
\hline F. SIALIDAE & & & & & $\mathrm{X}$ & & & & & \\
\hline F. GYRINI DAE & & & & $\mathrm{X}$ & & & & & & \\
\hline F. DYTISCIDAE & & $\mathrm{X}$ & & $\mathrm{X}$ & & & $\mathrm{X}$ & $\mathrm{X}$ & $\mathrm{X}$ & $\mathrm{X}$ \\
\hline F. HALI PLIDAE & & $\mathrm{X}$ & & & & $\mathrm{X}$ & & & & \\
\hline F. HYGROBIIDAE & & & $\mathrm{X}$ & & & & & & & \\
\hline F. ELMI DAE & $\mathrm{X}$ & $\mathrm{X}$ & $\mathrm{X}$ & $\mathrm{X}$ & $\mathrm{X}$ & & & $\mathrm{X}$ & $\mathrm{X}$ & $\mathrm{X}$ \\
\hline F. HYDROPTILIDAE & & & & $\mathrm{X}$ & & & & $\mathrm{X}$ & $\mathrm{X}$ & \\
\hline F. HYDROPSYCHIDAE & $\mathrm{X}$ & $\mathrm{X}$ & $\mathrm{X}$ & & $\mathrm{X}$ & $\mathrm{X}$ & $\mathrm{X}$ & $\mathrm{X}$ & $\mathrm{X}$ & $\mathrm{X}$ \\
\hline F. ECNOMIDAE & & & & & & & & $X$ & & \\
\hline F. MOLANNIDAE & $X$ & & & & & & & & & \\
\hline C PHRYGANEIDAE & $\mathrm{X}$ & & & & & & & & & \\
\hline F LEPTOCERIDAE & & & & & $\mathrm{X}$ & & & & & $\mathrm{X}$ \\
\hline F. LIMNEPHILIDAE & & & & & & $\mathrm{X}$ & & & & \\
\hline F. LIMONIIDAE & $\mathrm{X}$ & & & & & & & & & \\
\hline F. SIMULIIDAE & $\mathrm{X}$ & & & $\mathrm{X}$ & $\mathrm{X}$ & $X$ & & $\mathrm{X}$ & $\mathrm{X}$ & $\mathrm{X}$ \\
\hline F. CHIRONOMIDAE & $\mathrm{X}$ & $\mathrm{X}$ & $\mathrm{X}$ & $\mathrm{X}$ & $\mathrm{X}$ & $\mathrm{X}$ & $\mathrm{X}$ & $\mathrm{X}$ & $\mathrm{X}$ & $\mathrm{X}$ \\
\hline F. CERATOPOGI NIDAE & & & & & & & & $\mathrm{X}$ & & \\
\hline F. EMPIDIDAE & & & $X$ & & & & & & & \\
\hline
\end{tabular}


Tabla 4. Listado de taxa de macroinvertebrados que aparecen en el río Cigüela. Abreviaturas: L. Larvas; N: ninfas; A: adultos. List of macroinvertebrates' taxa found in the Cigüela River. Abbreviations: L. Larvae; N: nymphs; A: adults.

\begin{tabular}{|c|c|c|c|c|c|c|c|c|c|c|c|c|c|c|}
\hline \multirow{2}{*}{$\frac{\text { Momentos }}{\text { Puntos de muestreo }}$} & \multicolumn{7}{|c|}{1} & \multicolumn{7}{|c|}{2} \\
\hline & E1A & E1B & E2-E3 & E4 & E5 & E6 & E7 & E1A & E1B & E2-E3 & E4 & E5 & E6 & E7 \\
\hline Cl. OLIGOQUETOS & & & & & & & $\mathrm{X}$ & & & & & & $\mathrm{X}$ & $\mathrm{X}$ \\
\hline F. BRANCHIOBDELLIDAE & & & & & & & & & & & & & X & \\
\hline F. NAIDIDAE & & & & & & & & & & & & & & $\mathrm{X}$ \\
\hline F. AE LOSOMATIDAE & & & & & & $\mathrm{X}$ & $\mathrm{X}$ & & & & & & & \\
\hline F. VALVATI DAE & & & & & & & & & & & & & & $\mathrm{X}$ \\
\hline F. VIVIPARIDAE & & & & $\mathrm{X}$ & & & & & & & & & & \\
\hline F. HYDROBIIDAE & & & & & & & $\mathrm{X}$ & & & & & & & \\
\hline F. BYTHINELLIDAE & & & & & & & $\mathrm{X}$ & & & & & & & \\
\hline F. ANCYLIDAE & & & & & & & X & & & & & & $\mathrm{X}$ & $\mathrm{X}$ \\
\hline F. PHYSIDAE & $\mathrm{X}$ & & & & & $\mathrm{X}$ & X & & $\mathrm{X}$ & & & $\mathrm{X}$ & $\mathrm{X}$ & $\mathrm{X}$ \\
\hline F. LYMNAEIDAE & & & & $\mathrm{X}$ & & & X & & $\mathrm{X}$ & & $\mathrm{X}$ & & X & $\mathrm{X}$ \\
\hline F. SPHAERIIDAE & $\mathrm{X}$ & $\mathrm{X}$ & & $\mathrm{X}$ & & $\mathrm{X}$ & $\mathrm{X}$ & $\mathrm{X}$ & $\mathrm{X}$ & & & & $\mathrm{X}$ & $\mathrm{X}$ \\
\hline Cl. OSTRACODOS & $X$ & $\mathrm{X}$ & $X$ & $\mathrm{X}$ & & $\mathrm{X}$ & X & $\mathrm{X}$ & $\mathrm{X}$ & $\mathrm{X}$ & & & X & $\mathrm{X}$ \\
\hline O. HIDRACARINA & & $\mathrm{X}$ & $X$ & $\mathrm{X}$ & & & $\mathrm{X}$ & $\mathrm{X}$ & $X$ & $X$ & & & & $\mathrm{X}$ \\
\hline F. CAENIDAE & & $\mathrm{X}$ & & $\mathrm{X}$ & & & X & $\mathrm{X}$ & $\mathrm{X}$ & $\mathrm{X}$ & $\mathrm{X}$ & & $\mathrm{X}$ & $\mathrm{X}$ \\
\hline F. BAETIDAE & $\mathrm{X}$ & $\mathrm{X}$ & $X$ & $\mathrm{X}$ & X & $\mathrm{X}$ & $\mathrm{X}$ & $\mathrm{X}$ & $\mathrm{X}$ & $\mathrm{X}$ & $\mathrm{X}$ & $\mathrm{X}$ & $\mathrm{X}$ & $\mathrm{X}$ \\
\hline F. NEMOURIDAE & $\mathrm{X}$ & $\mathrm{X}$ & & & & & $\mathrm{X}$ & $\mathrm{X}$ & $\mathrm{X}$ & $\mathrm{X}$ & & & & \\
\hline F. CAPNIIDAE & & & & & & & $\mathrm{X}$ & & & & & & & \\
\hline F. LEUCTRIDAE & & $\mathrm{X}$ & & & & & & & & & & & & \\
\hline F. PERLODIDAE & $X$ & & & & $\mathrm{X}$ & & & $\mathrm{X}$ & & $\mathrm{X}$ & & & & \\
\hline F. PERLIDAE & & & $\mathrm{X}$ & & & & & & & & & & & \\
\hline F. CHLOROPERLIDAE & & $\mathrm{X}$ & & & & & & & & & & & & \\
\hline F. CALOPTERYGIDAE & $X$ & $\mathrm{X}$ & $\mathrm{X}$ & $\mathrm{X}$ & & $\mathrm{X}$ & $\mathrm{X}$ & $\mathrm{X}$ & & & $\mathrm{X}$ & & & \\
\hline F. PLATYCNEMIDIDAE & & & & & & & & & & & $X$ & & & \\
\hline F. COENAGIONIDAE & & & $X$ & & $\mathrm{X}$ & & $\mathrm{X}$ & & & & & $X$ & $X$ & \\
\hline F. HEBRIDAE & & & & & & & & & $\mathrm{X}$ & & & & & \\
\hline F. CORIXIDAE & & & & & & & & & $\mathrm{X}$ & & & & & \\
\hline O. MEGALOPTERES & & & & & & & & & & & & & & $\mathrm{X}$ \\
\hline F. SIALIDAE & & & & & $\mathrm{X}$ & & & $\mathrm{X}$ & & & $\mathrm{X}$ & $\mathrm{X}$ & & $\mathrm{X}$ \\
\hline F. GYRINIDAE & & & & & & & & & & $\mathrm{X}$ & & & & \\
\hline F. DYTISCIDAE & & & $\mathrm{X}$ & $\mathrm{X}$ & $\mathrm{X}$ & & & $\mathrm{X}$ & & $\mathrm{X}$ & $\mathrm{X}$ & & & $\mathrm{X}$ \\
\hline F. HALIPLIDAE & & & & $\mathrm{X}$ & & & & & & & & $\mathrm{X}$ & & \\
\hline F. HYDRAENIDAE & & & & & & $\mathrm{X}$ & & & & & & & & \\
\hline F. DRYOPIDAE & & & & & & $\mathrm{X}$ & & & & & & & & \\
\hline F. ELMIDAE & $\mathrm{X}$ & & $\mathrm{X}$ & & & $\mathrm{X}$ & $\mathrm{X}$ & & $\mathrm{X}$ & $\mathrm{X}$ & & $\mathrm{X}$ & $\mathrm{X}$ & $\mathrm{X}$ \\
\hline F. HYDROPTILIDAE & & $X$ & $X$ & X & & $\mathrm{X}$ & $\mathrm{X}$ & & $\mathrm{X}$ & & & $\mathrm{X}$ & & $\mathrm{X}$ \\
\hline $\begin{array}{l}\text { F. HYDROPSYCHIDAE } \\
\text { F. ECNMMIDAF }\end{array}$ & $\mathrm{X}$ & $\mathrm{X}$ & $\mathrm{X}$ & & & $\mathrm{X}$ & $\mathrm{X}$ & $\mathrm{X}$ & $\mathrm{X}$ & $\mathrm{X}$ & $\mathrm{X}$ & & $\mathrm{X}$ & $\mathrm{X}$ \\
\hline $\begin{array}{l}\text { F. ECNOMIDAE } \\
\text { F. POLYCENTROPODIDAE }\end{array}$ & & & & & & & $X$ & & & & & & $\mathrm{X}$ & \\
\hline $\begin{array}{l}\text { F. POLYCENTROPODIDAE } \\
\text { F. BERAEIDAE }\end{array}$ & & & $\mathrm{X}$ & $\mathrm{X}$ & $X$ & & $X$ & $\mathrm{X}$ & & & & $X$ & $X$ & $\mathrm{X}$ \\
\hline $\begin{array}{l}\text { F. BERAEIDAE } \\
\text { F. LIMONIIDAE }\end{array}$ & & & & & & & & & & $\begin{array}{l}X \\
X\end{array}$ & & & & \\
\hline F. CULICIDAE & & & $\mathrm{X}$ & & & & & & & & $\mathrm{X}$ & & $\mathrm{X}$ & \\
\hline F. SIMULIIDAE & $\mathrm{X}$ & $\mathrm{X}$ & $\mathrm{X}$ & & & $\mathrm{X}$ & $\mathrm{X}$ & $\mathrm{X}$ & $\mathrm{X}$ & & & & $X$ & $\mathrm{X}$ \\
\hline F. CHIRONOMIDAE & $\mathrm{X}$ & $\mathrm{X}$ & $\mathrm{X}$ & $\mathrm{X}$ & $X$ & $\mathrm{X}$ & $\mathrm{X}$ & $\mathrm{X}$ & $\mathrm{X}$ & $\mathrm{X}$ & $X$ & $X$ & $X$ & $\mathrm{X}$ \\
\hline F. CERATOPOGINIDAE & & $\mathrm{X}$ & & & & & $\mathrm{X}$ & & & & $\mathrm{X}$ & & & $\mathrm{X}$ \\
\hline F. EMPIDIDAE & & & & & & & $\mathrm{X}$ & & & $X$ & & & & $\mathrm{X}$ \\
\hline F. TABAN IDAE & & & $X$ & & & & & & & $\mathrm{X}$ & $\mathrm{X}$ & & & \\
\hline F. ANTHOMYIDAE & & & & & & $\mathrm{X}$ & $\mathrm{X}$ & & & & & & & $\mathrm{X}$ \\
\hline
\end{tabular}

(Odonata), y Phryganeidae, Leptoceridae, Limnephilidae y Molanniade (Tricoptera), y en el Cigüela: Leuctridae y Chloroperlidae (Plecoptera), Hebridae y Corixidae (Heteroptera), y Beraeidae (Tricoptera). Hay familias que se encuentran en todos los puntos de ambos ríos independientemente del tipo de sustrato: Gammaridae (Amphipoda), Baetidae (Ephemeroptera) y Chironomidae (Diptera). En el río Valdepineda además de las anteriores, también se encuentran las familias Coenagrionidae (Odonata), Hidropsychidae (Tricoptera) y Lymnaeidae (Mollusca). La mayoría de ellas corresponden a estados inmaduros de insectos alcanzando en el río Cigüela el $76 \%$ y en el río Valdepineda el $79 \%$ del total.

En cuanto a la afección de la obra de zanja, no cabe destacar un cambio relevante en el número $\mathrm{y}$ en la presencia de taxa una vez realizada la 
Tabla 5. Clases de calidad según el índice BMWP' en cada una de las estaciones muestreadas en ambos ríos antes y después de la obra. $B M W P$ ' rankings at each sampling site from both rivers before and after construction.

\begin{tabular}{lccccccc}
\hline Río Cigüela & E1A & E1B & E2-E3 & E4 & E5 & E6 & E7 \\
\hline $\begin{array}{l}\text { Antes obra } \\
\text { Después obra }\end{array}$ & II & II & II & III & III & II & I \\
\hline Río Valdepineda & A1A & A1B & A2-A3 & A4 & A5 & & \\
\hline Antes obra & I & II & III & III & II & & \\
Después obra & II & III & I & II & I & & \\
\hline
\end{tabular}

obra (Tablas 3 y 4). Tampoco se registran cambios relevantes en lo que al índice BMWP' (Tabla 5) se refiere. Este índice sólo muestra una disminución en la clase de calidad de las aguas en la última estación del río Cigüela (E7) aguas abajo de la confluencia de los dos ríos. En el río Valdepineda las clases de calidad de las aguas disminuyen ligeramente en las dos estaciones aguas arriba de las obras y aumentan en las estaciones situadas aguas abajo.

Respecto a la ictiofauna de ambos ríos, en el periodo de construcción de la zanja, cabe señalar que no se pescó ningún ejemplar en ninguna de las estaciones muestreadas. La causa más probable de este resultado nulo puede que sea la baja temperatura del agua de los dos ríos, alrededor de $3^{\circ} \mathrm{C}$ (la capa superficial del agua se encontraba helada). La baja temperatura del agua, reduce la conductividad disminuyéndose la capturabilidad de los peces. La temperatura influye, además sobre el estado metabólico de los peces de manera que al disminuir, se reduce la tasa metabólica. Este fenómeno ha sido descrito a temperaturas bajas (Lobón-Cerviá, 1991). Se descarta que se deba a un desplazamiento temporal de los individuos a otros tramos no afectados por la obra ya que los muestreos se realizaron con varios días de diferencia con la actividad sobre el lecho de los ríos. De todas maneras, se ha considerado interesante avanzar los resultados parciales obtenidos en otra etapa posterior del proyecto, la fase de rehabilitación, dado lo llamativo de los resultados obtenidos y la singularidad de la fauna íctica encontrada en estos ríos, una fauna rica, estructurada en una comunidad típica de ríos mediterráneos. Así, podemos citar la presencia de las siguientes especies: la colmilleja (Cobitis paludica), la pardilla (Chondrostoma lemmingii), el barbo mediterráneo (Barbus guiraonis), el barbo cabecicorto (Barbus microcephalus), el calandino (Squalius alburnoides) y el cacho (Squalius pyrenaicus). Del mismo modo se destaca la ausencia de especies alóctonas, como el blackbass (Micropterus salmoides), el lucio (Esox lucius) y el percasol (Lepomis gibbosus) que habitan aguas abajo en las lagunas de Ruidera.

$\mathrm{Si}$ observamos los datos en conjunto para las tres estaciones de muestreo (Tabla 6), captura-

Tabla 6. Número de individuos de las diferentes especies de peces capturados en los ríos Valdepineda y Cigüela antes y después de la construcción. Los valores significativos de chi cuadrado están marcados con asterisco. Number of specimens of the different fish species captured in the Valdepineda and Ciguela Rivers before and after construction. Significant chi square values are marked with an asterisk.

Colmilleja* Pardilla* Cacho* Calandino* $\begin{gathered}\text { Barbo } \\ \text { mediterráneo }\end{gathered} \begin{gathered}\text { Barbo } \\ \text { cabecicorto }\end{gathered}$

\begin{tabular}{llllllll}
\hline \multirow{2}{*}{$\begin{array}{l}\text { P1 } \\
\text { Río Cigüela }\end{array}$} & Antes obra & 1 & 1 & 2 & 11 & 0 & 0 \\
\hline & Después obra & 1 & 2 & 18 & 2 & 0 & 0 \\
\hline $\begin{array}{l}\text { P2 } \\
\text { Río Valdepineda }\end{array}$ & Antes obra & 22 & 4 & 0 & 2 & 1 & 0 \\
\hline & Después obra & 58 & 24 & 0 & 2 & 0 & 0 \\
\hline $\begin{array}{l}\text { P3 } \\
\text { Confluencia }\end{array}$ & Antes obra & 0 & 0 & 3 & 6 & 0 & 0 \\
\hline & Después obra & 0 & 4 & 6 & 2 & 1 \\
\hline
\end{tabular}


mos un mayor número de ejemplares, de 5 de las 6 especies presentes, después de la realización de la fase de rehabilitación con la retirada de las pistas de trabajo. La colmilleja es la especie más abundante, se han capturado 23 ejemplares antes de la rehabilitación y 59 después. La pardilla le sigue en abundancia con 5 ejemplares antes de la rehabilitación y 30 después. Estas dos especies son más abundantes en la estación P2 (río Valdepineda). En el mismo orden hemos capturado cachos, especie ausente en la estación P1 del río Cigüela, 5 ejemplares antes de la rehabilitación y 24 después. En cuanto a los calandinos, es la única especie cuyo número de ejemplares ha disminuido después de la rehabilitación, con 15 ejemplares capturado antes y 9 después. Aunque el número no sea significativo, cabe señalar la presencia de 1 ejemplar de barbo mediterráneo y otro de barbo cabecicorto después de la retirada de la pista de trabajo. Comparando la frecuencia de distribución antes y después de la obra, encontramos diferencias significativas en la abundancia de la colmilleja $\left(\chi^{2}=58.909 ; \mathrm{p}<0.001\right)$, la pardilla $\left(\chi^{2}=101\right.$; $\mathrm{p}<0.001)$, el cacho $\left(\chi^{2}=131 ; \mathrm{p}<0.001\right)$ y el calandino $\left(\chi^{2}=15.864 ; \mathrm{p}<0.001\right)$.

\section{DISCUSIÓN}

Los impactos potenciales de la construcción de gasoductos en los cruces hídricos son, fundamentalmente, el incremento y deposición de los sólidos en suspensión movilizados durante la ejecución de las obras, la barrera para la fauna que produce la instalación de la pista de trabajo, la alteración de la morfología del cauce en el área directamente afectada por la obra, y el vertido accidental de aceites y materiales de construcción. De todos ellos, el que produce un mayor efecto directo e inmediato sobre el medio es el incremento de las cargas de sedimentos producidos por la construcción en el propio lecho del río y las actividades constructivas que producen erosión de los suelos expuestos a lo largo de la pista de trabajo. La deposición de estos sedimentos aguas abajo del punto de cruce afecta al hábitat de la flora acuática, del macrobentos y de los peces al rellenar los intersticios del sustrato, reduciendo el flujo de agua que lo atraviesa, desencadenando bajas en la producción béntica, y aumentando la mortalidad de los huevos o de las larvas de peces. Por otra parte, el incremento de los sólidos en suspensión reduce la penetración de la luz y disminuye la producción primaria (Reid y Anderson, 1999).

La construcción de la zanja en el punto de cruce modifica radicalmente el hábitat en este tramo del río al alterar la morfometría natural del canal y la granulometría del lecho (Petts y Foster, 1985). Las alteraciones en la morfología del canal producen, además, cambios en la velocidad de la corriente con efecto sobre los tramos situados aguas arriba y abajo del punto de cruce, aunque no se han encontrado diferencias en los caudales antes y después de la obra.

En cuanto a los sólidos en suspensión, su desplazamiento se ve dificultado por el escaso caudal que circula por los cauces, y por la abundante vegetación acuática del lecho del río y ribera, que contribuye a retener las partículas en suspensión. Los resultados observados muestran que durante los primeros 100 metros aguas abajo del punto de cruce se están depositando la mayor parte de los sedimentos que la obra de construcción de la zanja ha movilizado en el río. Las diferencias significativas en la composición granulométrica de los sedimentos del lecho del río en las estaciones E1A, E1B, E5, A5 y E6 obtenidas, no parecen explicarse teniendo en cuenta las valores de los sólidos en suspensión obtenidos durante la fase de ejecución de la obra. Hay que tener en cuenta que la microtopografía del cauce influye notablemente en la granulometría que existe en el lecho del río, lo que explicaría la gran variabilidad obtenida entre las réplicas de las muestras tomadas, como se puede ver en los altos coeficientes de variación. La precisión de esta metodología mejoraría incrementando el número de réplicas por punto de muestreo tomadas aleatoriamente en lugares homogéneamente parecidos.

Por otra parte, se espera que los mayores impactos derivados del desarrollo de la obra 
sobre las comunidades de macroinvertebrados se produzcan en las estaciones más cercanas aguas abajo del punto de cruce, mediante la modificación en el número total de familias como consecuencia de un incremento en la deriva de macroinvertebrados durante la fase de construcción, tal y como Kraft (1981) y Tsui y McCart (1981) han observado en sus estudios, e incluso con la aparición o desaparición de las familias más sensibles al efecto de los sólidos en suspensión o a la destrucción de hábitats. Sin embargo, esta tendencia no se observa en los resultados presentados en el presente estudio.

Por tanto, y con los resultados que hemos obtenido no podemos concluir que exista una clara afección de la obra sobre los diferentes taxa de macrobentos y por ende, en el índice de calidad de las aguas BMWP'. Tampoco en otros estudios ha sido posible demostrar cambios en la estructura de las comunidades aguas abajo de la obra (Petersen et al., 1978; Kraft, 1981), aunque sí se ha encontrado una reducción en la densidad del macrobentos (Kraft, 1981; Tsui y McCart, 1981; Anderson et al., 1998).

Hemos de considerar que, a pesar de encontrarnos en el tramo alto de estos dos ríos, ambos discurren por una zona muy antropizada donde se desarrollan unas prácticas agrícolas que aumentan la erosión y la carga de sólidos en suspensión en la columna de agua. Debido a su baja pendiente, se produce un aumento en la deposición de sedimentos en el sustrato. Por tanto, es muy probable que las comunidades florísticas y faunísticas que lo habitan estén adaptadas a estos cambios.

Otro impacto a tener en cuenta es la barrera temporal para el paso de fauna que causa el establecimiento de una pista de trabajo, perpendicular a la dirección de la corriente y las derivaciones del caudal del río (by-pass) que se realizan durante la construcción. En los resultados se observa un claro aumento de los ejemplares capturados después de haber sido retirada la pista de trabajo y una diferencia significativa en su frecuencia de distribución, lo que sugiere que la obra está produciendo un efecto barrera que hay que tener en cuenta a la hora de planificar el trabajo y de diseñar las estructuras de drenaje (vainas), de forma que no se obstaculice el acceso de la ictiofauna al tramo superior del río.

\section{AGRADECIMIENTOS}

Agradecemos la ayuda en los trabajos de campo a Carmen Belén Díaz, Ángel Gutiérrez, Helena Palacios, Paloma Pinilla e Inés Iribarren, y la colaboración de Ignacio Doadrio, José Ambrosio González, Luis Garcerán y Cimera, S.A. Este proyecto se enmarca dentro del convenio de colaboración entre la Dirección General de Calidad y Evaluación Ambiental del Ministerio de Medio Ambiente y el Instituto Geológico y Minero de España ( $n^{\circ}$ de Sicoan: 200050).

\section{BIBLIOGRAFÍA}

ANDERSON, P., C. FRAIKIN \& T. CHANDLER. 1998. Natural gas pipeline crossing of a coldwater stream: Impacts and Recovery. Proceedings of the International Pipeline Conference. Calgary. Alberta.

ANDERSON, P., B. TAYLOR \& G. BALCH. 1996. Quantifying the effects of sediment release on fish and their habitats. Canadian Manuscript Report of Fisheries and Aquatic Sciences, 2346. 110 pp.

ALBA-TERCEDOR, J. y A. SÁNCHEZ-ORTEGA. 1988. Un método rápido y simple para evaluar la calidad biológica de las aguas corrientes basado en el de Hellawell (1978). Limnetica, 4: 51-56

BARTON, B. 1977. Short-term effects of highway construction on the limnology of a small stream in southern Ontario. Freshwat. Biol., 7: 99-108.

CATALÁN LA FUENTE, J. 1981. Química del agua. Talleres Gráficos Alonso, S.A., Madrid.

CORDONE, A. \& D. KELLEY. 1961. The influences of inorganic sediment on the aquatic sediment on the aquatic life of streams. California Fish and Game, 47: 189-228.

CHUTTER, F. 1969. The effects of silt and sand on the invertebrate fauna of streams and rivers. Hydrobiologia, 34: 57-76.

FISRWG, 1998. Stream Corridor Restoration: Principles, Processes and Practices. Federal Interagency Stream Restoration Working Group. 
GPO Item No. 0120-A; Su Docs Nº. A 57.6/2: EN 3/PT.653 pp.

KRAFT, L. 1981. Aquatic systems instigations in relation to the Alaska Highway gas pipeline crossings of the Bow and Red Deer Rivers, Alberta, 1980. Vol. 1. Report prepared by Environmental Management Associates.

LOBÓN-CERVIÁ, J. 1991. Dinámica de poblaciones de peces en ríos. Pesca eléctrica y métodos de capturas sucesivas en la estima de abundancias. Monografías del Museo Nacional de Ciencias Naturales. CSIC. Madrid. 156 pp.

MCKINNON, G. \& F.N. HNYTKA. 1988. The effect of winter pipeline construction on the fishes and fish habitat of Hodgson Creek, NWT. Canandian Technichal Report of Fisheries and Aquatic Sciences, 1598.

NEWCOMBE C. \& J. JENSEN. 1996. Channel suspended sediment and fisheries: a synthesis for quantitative assessment of risk and impact. North
American Journal of Fisheries Management, 16: 693-727.

NEWCOMBE, C. \& D. MACDONALD. 1991. Effects of suspended sediments on aquatic ecosystems. North American Journal of Fisheries Management, 11: 72-82.

PETERSEN, G., G. SMITH \& L. BODNARUK. 1978. Some short term effects of experimental trenching on two streams in the Caribou River Drainage, Manitoba, 1977. Polar Gas Project.

PETTS, G. \& I. FOSTER. 1985. Rivers and Landscape. Ed. Edward Arnold. London.

REID, S.M. \& P.G. ANDERSON. 1999. Effects of sediment released during opencut pipeline water crossings. Canadian Water Resources Journal 24(3): 235-261.

TSUI, P. \& P. MCCART. 1981. Effects of stream crossing by a pipeline on the benthic macroinvertebrate communities of a small mountain stream. Hydrobiologia, 79: 271-276. 